\title{
Symmetrization Method of AC Traction Substation with Power Balancer
}

\author{
Martin Pittermann \\ Department of Power Electronics and \\ Machines \\ University of West Bohemia \\ Pilsen, Czech Republic \\ pitterma@fel.zcu.cz
}

\author{
Milos Straka \\ Research and Innovation Centre for \\ Electrical Engineering \\ University of West Bohemia \\ Pilsen, Czech Republic \\ strakami@fel.zcu.cz
}

\author{
Vojtech Blahnik \\ Research and Innovation Centre for \\ Electrical Engineering \\ University of West Bohemia \\ Pilsen, Czech Republic \\ lucke@fel.zcu.cz
}

\begin{abstract}
The paper describes the symmetrization method of power converter used in topology of AC traction substation with power balancer. This topology provides the three-phase current symmetrization and the reactive power compensation for AC traction substation feeding $25 \mathrm{kV} / 50 \mathrm{~Hz}$ traction catenary. The described symmetrization method is based on principles of the Steinmetz symmetrizing circuit. The main paper contribution is a derivation and description of the symmetrization equations, which are used for the control algorithm of multilevel semiconductor converters in a delta connected circuit.
\end{abstract}

Keywords- symmetrization method; Steinmetz; current symmetrization; traction substation

\section{INTRODUCTION}

The AC traction grid in the Czech Republic (single phase $25 \mathrm{kV} / 50 \mathrm{~Hz}$ ) is fully feed from a distribution power grid. This connection is provided through the traction substations, which are handled in open delta configuration, for particular symmetrization of consumed power from the distribution power grid. However, the open delta configuration does not ensure full symmetrization, for these reasons new advanced TSS topologies are being tested in the Czech Republic. One perspective topology is traction substation with electronic balancer introduced in [1] and [2].

This paper deals with the AC TSS (traction substation) with electronic balancer. The main part of this paper is concern to derivation of the symmetrization method and equations required for the balancer unit. An extension of important knowledge for the already known topology and for the advanced topology, were presented in [1], [2], [3], [4], [5].

The first part of the paper describes common symmetrization method (Steinmetz theory) and defines the way in which the method for a controlled electronic balancer can be used (introduced in [2]). The second part of the paper describes the derivation of equations used for symmetrization and compensating of single-phase reactive load. The resulting equations are used for the control algorithm of the traction substation with electronic balancer and were tested on simulation model. The results of the current behavior are listed in the last section of this article. They are used to verify the proposed method of symmetrization.

The connection of introduced traction substation with the electronic balancer is shown in Fig. 1. This topology is perspective solution because it is in interest to major industrial manufacturers, as is shown in [3] and [4] (ABB and Siemens). The main advantage of this solution is the possibility of TSS working even in case of failure of balancer (of course without the symmetrization).

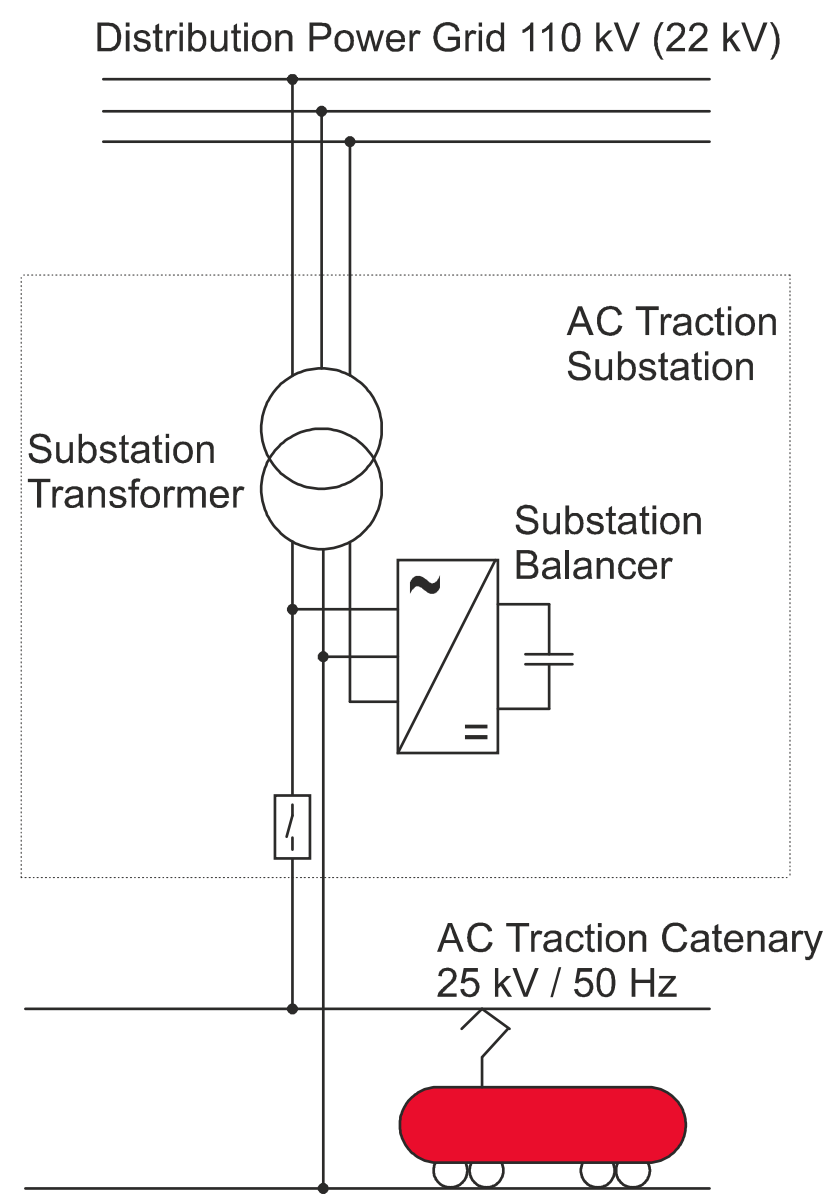

Fig. 1. Ilustration of connected distribution grid and AC traction catenry via traction substation with electronic balancer

\section{STEINMETZ SYMMETRIZING CIRCUIT}

The power symmetrization derived for the traction substation is based on common principles of Steinmetz symmetrizing circuit. This circuit is shown in Fig. 2, which is the delta connection of circuit with single phase of active load (R) and symmetrizing reactive components $(\mathrm{L}, \mathrm{C})$ in other two. Power symmetrization is based on finding the correct values of the passive reactive components $\mathrm{L}$ and $\mathrm{C}$. Balancing of input currents i1, i2, i3 is provided by oscillation of symmetrizing energy between reactive element $L$ and reactive element C. [6] [7] These oscillating currents $\left(i_{R}, i_{L}, i_{C}\right)$ are called circulating currents and their value depends on the active power of the load (R). [8] 


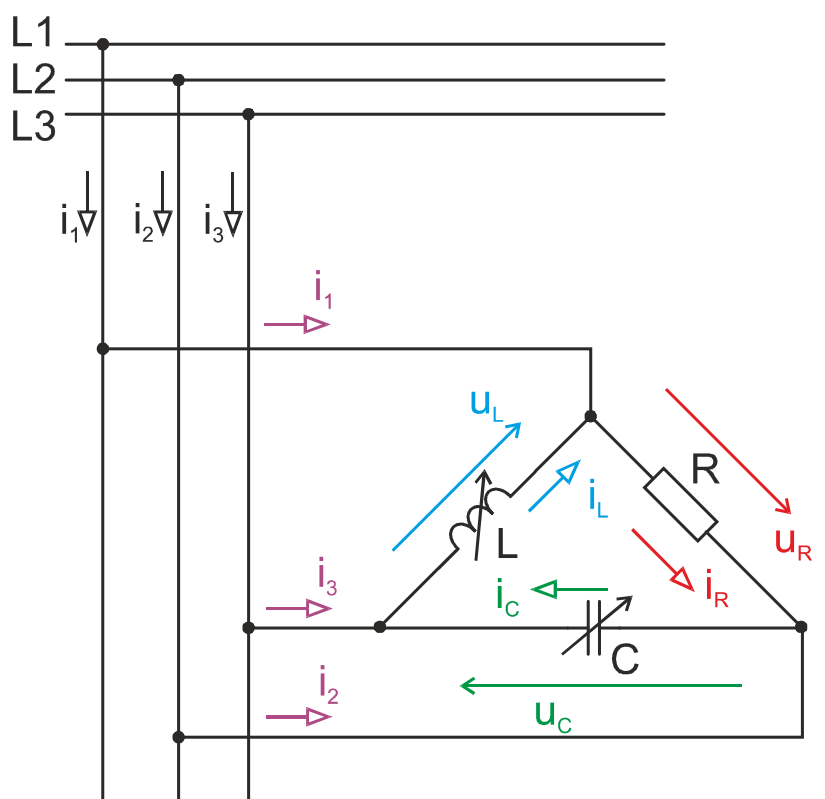

Fig. 2. General electrical circuit diagram for the Steinmetz symmetrizing method

This principle can be conveniently viewed on the illustrative phasor diagram in Fig. 3.

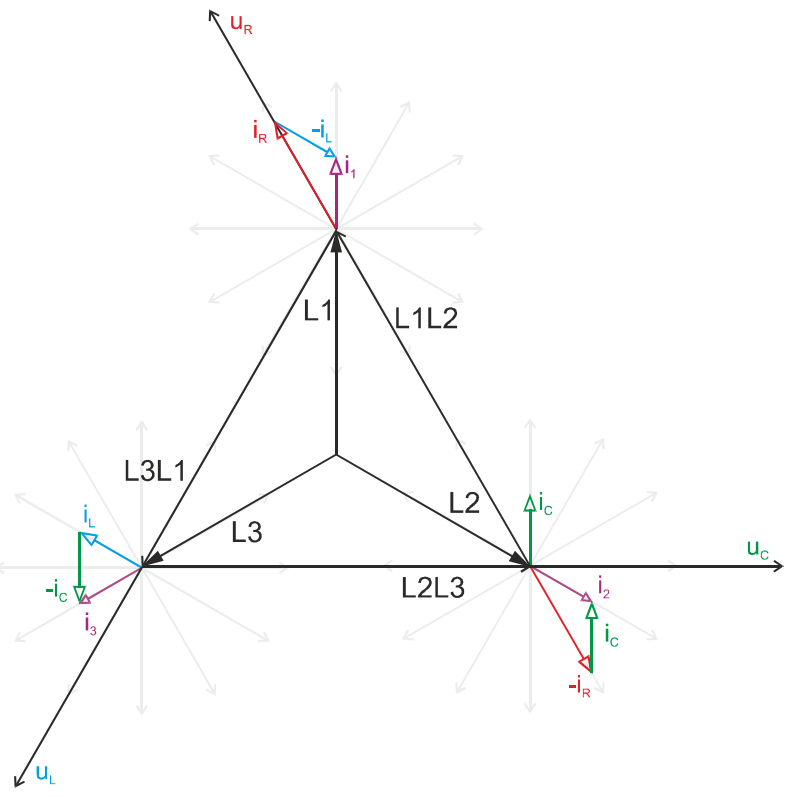

Fig. 3. The phasor diagram coresponding with the princeple of Steinmetz symmetrizing method

Steinmetz circuit is able to mitigate voltage unbalance as is described in [8].

\section{SYMMETRIZING OF SIGLE PAHSE TRACTION CATENARY}

The principle of symmetrization described in chapter II is necessary to develop in case of reactive load of traction substation. For older locomotives, we assume the possibility of an RL load (thyristor converter at input side). For this case is possible to redraw the symmetrizing circuit into the form shown in Fig. 4.
Inductor $\mathrm{L}_{\mathrm{bal}}$ and Capacitor $\mathrm{C}_{\mathrm{bal}}$ are used for symmetrizing and Capacitor $\mathrm{C}_{\mathrm{FKZ}}$ is used for handling of compensation of the reactive power of the load.

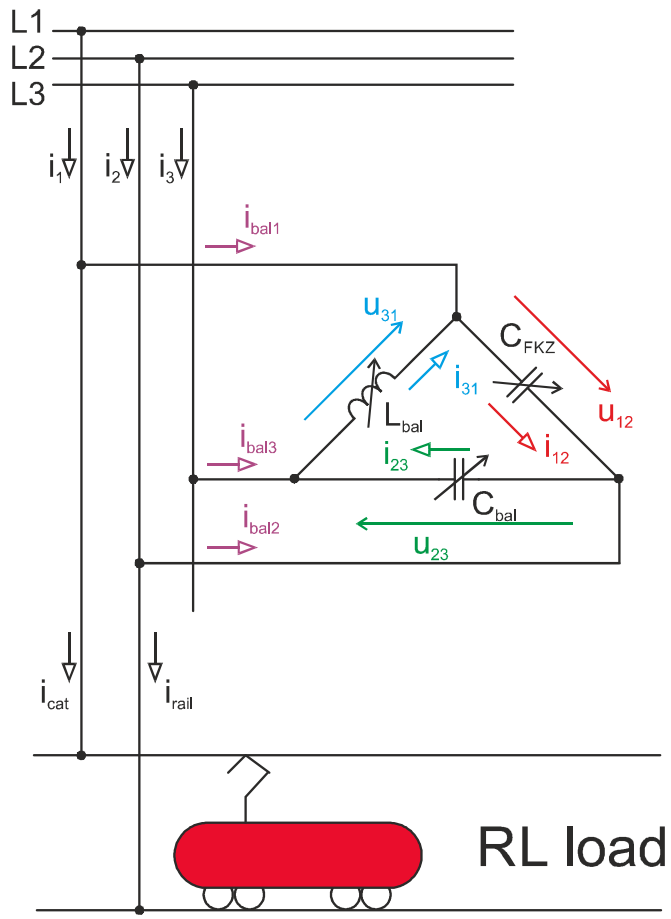

Fig. 4. The modified electrical circuit diagram of symmetrizing method method used for AC traction substation with electronic balancer

The power circuit of traction substation with electronic balancer is shown in Fig. 5. The electronic balancer is realized by multilevel converter based on CHB (Cascade H-Bridge) technology. The currents for symmetrization and compensation are $i_{\text {bal12 }}, i_{\text {bal23 }} i_{\text {bal31 }}$. These currents are the most important for us, because there are used as the referential currents for the control algorithm. By controlling of these currents, we are therefore influencing the currents drawn from the distribution power grid.

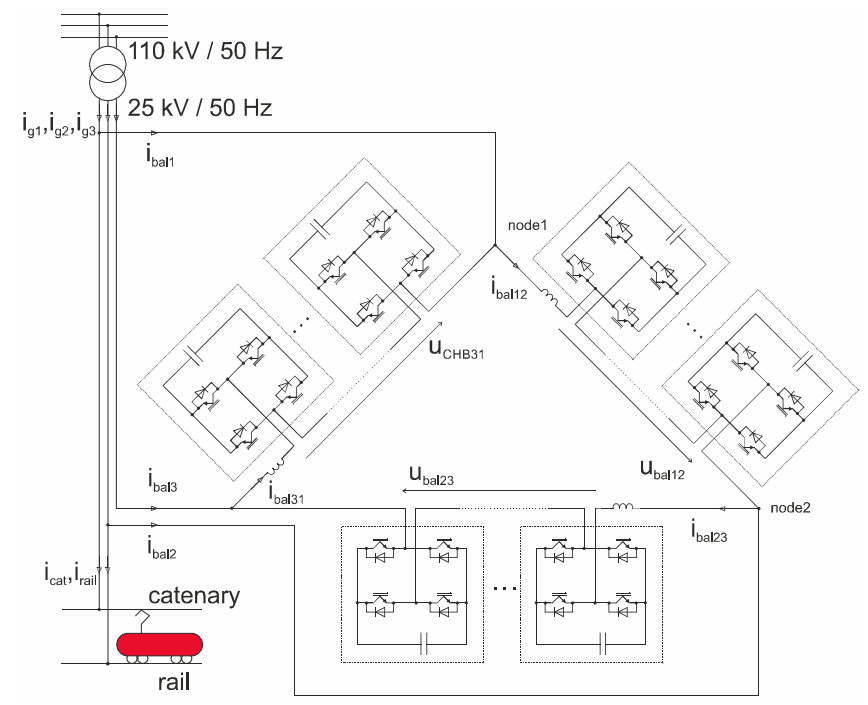

Fig. 5. Practical realisation of proposed TSS topology 
Equation (1) describes the incoming power, from the distribution power grid ( $\left.\mathrm{P}_{\text {input_grid }}\right)$, by phase voltage $\mathrm{u}_{\mathrm{L}}$ and current $i_{L}$. The output power from TSS for catenary ( $\left.P_{\text {input_cat }}\right)$ is given by (2).

$$
\begin{aligned}
& P_{\text {input_grid }}=3 \cdot u_{L} \cdot i_{L}=\left(\sqrt{3} \cdot u_{L}\right) \cdot\left(\sqrt{3} \cdot i_{L}\right) \\
& P_{\text {input_cat }}=u_{\text {cat }} \cdot i_{\text {cat }}=\sqrt{3} \cdot u_{L} \cdot i_{\text {cat }}
\end{aligned}
$$

If are the equation (1) and (2) equals (i.e. $\mathrm{P}_{\text {input_grid }}=$ $\mathrm{P}_{\text {input_cat }}$ ), for ideal power factor $\mathrm{PF}=1$ (only active power of the load or compensated load) then apply:

$$
i_{\text {cat }}=-i_{\text {rail }}=\sqrt{3} \cdot i_{L}
$$

In case branch 12 only takes care of compensation of reactive power in catenary (a large number of older locomotives are still operating with a diode rectifier or with a controlled thyristor rectifier in the Czech Republic), it is possible to describe active current by equation (4), resp. reactive current by equation (5) and by actual phase shift of catenary voltage $(\vartheta=\omega t)$ :

$i_{c a t_{-} a c t i v}=I_{m_{-} c a t_{-} a c t i v} \cdot \cos (\vartheta)$

$$
i_{\text {cat_reactiv }}=I_{m_{-} \text {cat_reactiv }} \cdot \cos \left(\vartheta-\frac{\pi}{2}\right)
$$

It is possible to compute input currents of the balancer as difference of wanted current and catenary current $\left(\mathrm{i}_{\mathrm{bal}}=\mathrm{i}_{\mathrm{w}_{\mathrm{L}} \mathrm{L}^{-}}\right.$ $i_{\text {cat_activ }} i_{\text {cat_reactiv }}$ ). First branch of balancer (branch 12) compensate the reactive power of current.

$$
\begin{aligned}
& i_{\text {bal1 }}=i_{w_{-} L 1}-i_{\text {cat_activ }}-i_{\text {cat_reactiv }} \\
& i_{\text {bal1 }}=\frac{1}{\sqrt{3}}\left(I_{m_{-} \text {cat_activ }} \cdot \cos \left(\vartheta-\frac{\pi}{6}\right)\right)-\left(I_{m_{-} \text {cat_activ }} \cdot \cos (\vartheta)\right)-\left(I_{m_{-} \text {cat_reactiv }} \cdot \cos \left(\vartheta-\frac{\pi}{2}\right)\right)
\end{aligned}
$$

$$
i_{\text {bal } 2}=i_{w_{-} L 2}-i_{c a t_{-} a c t i v_{-} L 2}-i_{c a t t_{-} \text {reactiv } L 2}=i_{w_{-} L 2}+i_{\text {cat_activ } L 2}+i_{\text {cat_reactiv } L 2}
$$$$
i_{\text {bal } 2}=\frac{1}{\sqrt{3}}\left(I_{m_{-} \text {cat_activ }} \cdot \cos \left(\vartheta-\frac{5 \pi}{6}\right)\right)+\left(I_{m_{-} c a t \_a c t i v} \cdot \cos (\vartheta)\right)+\left(I_{m_{-} \text {cat_reactiv }} \cdot \cos \left(\vartheta-\frac{\pi}{2}\right)\right)
$$

$$
i_{\text {bal } 3}=i_{w_{-} L 3}-i_{c a t_{-} a c t i v_{-} L 3}-i_{\text {cat_reactiv_ } L 3}=i_{w_{-} L 3}-0-0
$$$$
i_{\text {bal } 3}=\frac{1}{\sqrt{3}}\left(I_{m_{-} c a t_{-} a c t i v} \cdot \cos \left(\vartheta+\frac{\pi}{2}\right)\right)
$$

The derivation of resulting equations for the balancer branches $(12,23,31)$ is based on Kirchhoff's law and on the assumption, that branch 12 compensate the reactive power only:

$i_{\text {bal12 }}=-I_{m_{-} \text {cat_reactiv }} \cdot \cos \left(\vartheta-\frac{\pi}{2}\right)$

First Kirchhof's law applied for node 2 (i.e. equation for currents $\mathrm{i}_{\text {bal23 }}=\mathrm{i}_{\text {bal12 }}+\mathrm{i}_{\text {bal2 }}$ ) brings the equation (10):

$i_{\text {bal } 23}=\frac{1}{\sqrt{3}}\left(I_{m_{-} \text {cat_activ }} \cdot \cos \left(\vartheta-\frac{5 \pi}{6}\right)\right)+\left(I_{m_{-} \text {cat_activ }} \cdot \cos (\vartheta)\right)$

Node 1 yields the equations $i_{\text {bal31 }}=i_{\text {bal12 }}-i_{\text {bal1 }}$ from which can be formulate:

$i_{\text {bal31 }}=-\frac{1}{\sqrt{3}}\left(I_{m_{-} c a t \_a c t i v} \cdot \cos \left(\vartheta-\frac{\pi}{6}\right)\right)+\left(I_{m_{-} c a t_{-} a c t i v} \cdot \cos (\vartheta)\right)$

The results derived from the equations (9) (10) (11) intended for balancer control were tested in simulation model. The load nominal value was $10.8 \mathrm{MW}$ of the active power and 6,25 MVAr of the reactive power. The steady state of catenary current is captured in Fig. 6. where the current is lagged by $\pi / 6 \mathrm{rad}$.

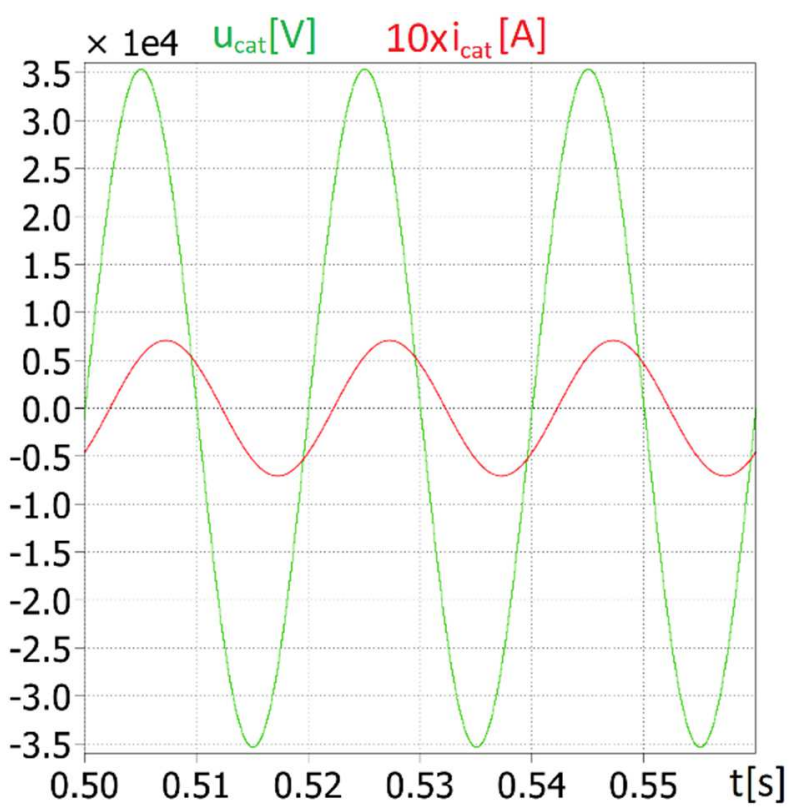

Fig. 6. Simulation model output - Voltage and current at catenary side with rective power 
Symmetrical currents drawn from the distribution power grid are shown in Fig. 7.

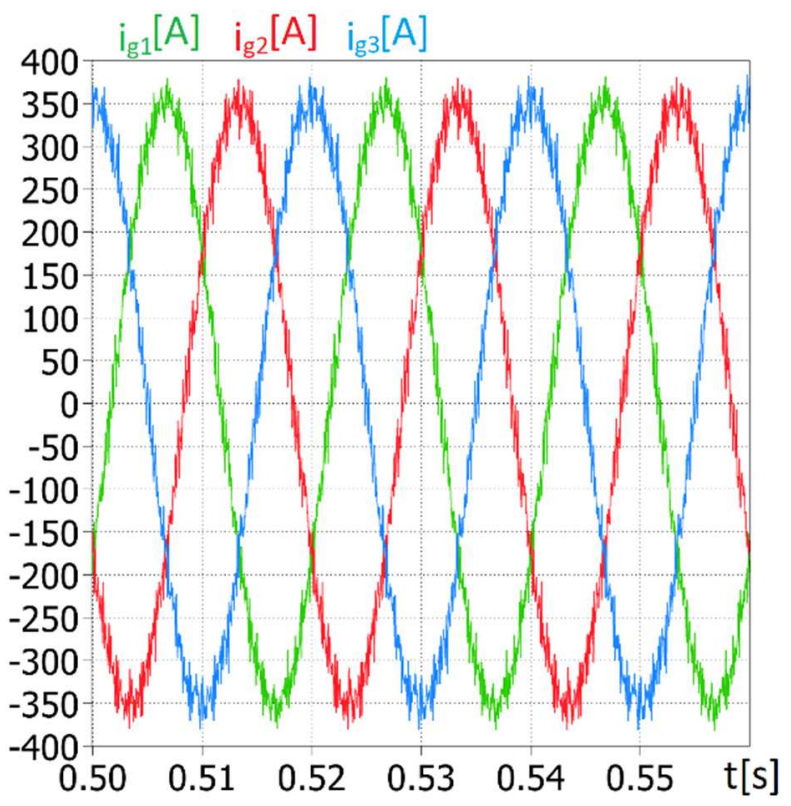

Fig. 7. Simulation model output - Currents at grid sidefully symetrized

The currents of individual branches of the balancer unit are shown in Fig. 8. These currents are calculated by formulas (9), (10), (11).

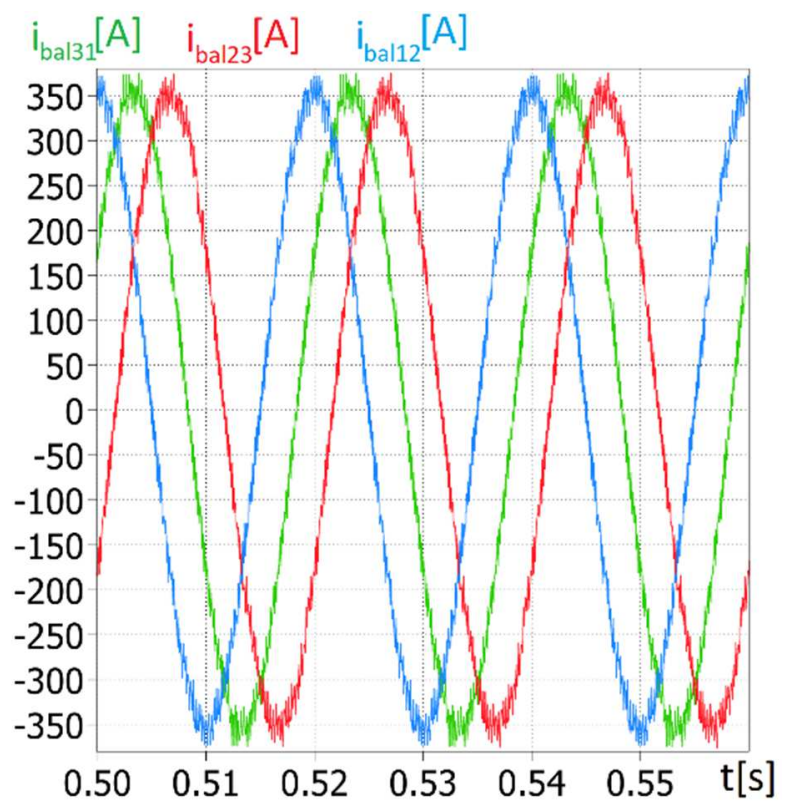

Fig. 8. Simulation model output - Electronic balancer currents at delta connected circuit

\section{CONCLUSION}

The three phase symmetrization method for single phase load (AC traction substation feeding $25 \mathrm{kV} / 50 \mathrm{~Hz}$ traction catenary) was derived. The symmetrization method is based on the Steinmetz's symmetrizing circuit. This principle is extended for the electronic balancer in delta connection. The resulting equations (9), (10), (11) (final balancing equations) are important for using at control of symmetrization unit in delta connection as is a presented in [10]. However, there is also possible to find the other interesting solution without balancer unit, how is presented in [11]. The results are used for development work for the design of new a TSS topology, developed in collaboration with industrial partner, for use in new TSS in the Czech Republic. The symmetrization balancer is able to complemented by device used for the controlled cooperation of several TSSs to a common traction line.

\section{ACKNOWLEDGMENT}

This research has been supported by the Ministry of Education, Youth and Sports of the Czech Republic under the project OP VVV Electrical Engineering Technologies with High-Level of Embedded Intelligence CZ.02.1.01/0.0/0.0/18_069/0009855 and under project SGS-2021-021.

\section{REFERENCES}

[1] V. Blahnik, M. Straka and M. Pittermann, "Application of Power Electronic Converter for Actual Topologies AC Traction Substation," 2020 International Conference on Applied Electronics (AE), Pilsen, Czech Republic, 2020, pp. 1-4

[2] V. Blahnik, M. Straka and M. Pittermann, "Control of Multilevel Converter for AC Traction Substation with Power Symmetrization Unit," 2020 International Conference on Applied Electronics (AE), Pilsen, Czech Republic, 2020, pp. 1-4.

[3] R. Grünbaum, "SVC for the Channel Tunnel rail link: Providing flexibility and power quality in rail traction," IEE Seminar on Power it's a Quality Thing, London, 2005, pp. 1-2.

[4] R. Gruber, D. O’Brien, " Use of Modular Multilevel Converter (MMC) Technology in Rail Electrification AusRAIL 2014, Perth Australia, May 2015.

[5] Serrano-Jiméneza, L. Abrahamssonb, S.Castano-Solísc, J.Sanz-Feitoa, "Electrical railway power supply systems: Current situation and future trends," International Journal of Electrical Power \& Energy Systems, vol. 92, pp. 181-192, November 2017.

[6] L. Sainz, L. Monjo, S. Riera and J. Pedra, "Study of the Steinmetz Circuit Influence on AC Traction System Resonance," in IEEE Transactions on Power Delivery, vol. 27, no. 4, pp. 2295-2303, Oct. 2012, doi: 10.1109/TPWRD.2012.2211084.

[7] L1. Monjo, L. Sainz, S. Riera \& J. Bergas (2013) Theoretical Study of the Steinmetz Circuit Design, Electric Power Components and Systems, 41:3, 304-323, DOI: 10.1080/15325008.2012.742944

[8] M. Yao, I. A. Hiskens and J. L. Mathieu, "Applying Steinmetz Circuit Design to Mitigate Voltage Unbalance Using Distributed Solar PV," 2019 IEEE Milan PowerTech, 2019, pp. 1-6, doi: 10.1109/PTC.2019.8810692.

[9] A. Steimel, Electric traction - motive power and energy supply: basics and practical experience, 2nd edition. München, germany: DIV Deutscher Industrieverlag GmbH, 2014. ISBN 978-3-8356-7257-4

[10] V. Blahnik, M. Pittermann, M. Straka, "Operating States of Load Balancer for AC Traction Substation Based on CHB Topology", 19th IEEE-PEMC, Gliwice, Poland 2021

[11] M. Tanta, J.G. Pinto, V.Monteiro, A.P. Martins, A.S. Carvalho, J.L. Afonso, "Topologies and Operation Modes of Rail Power Conditioners in AC Traction Grids: Review and Comprehensive Comparison" Energies 13, no. 9: 2151. 